\title{
High disease impact of myotonic dystrophy type 2 on physical and mental functioning
}

\author{
Alide A. Tieleman • Kathleen M. Jenks • \\ Joke S. Kalkman • George Borm • \\ Baziel G. M. van Engelen
}

Received: 6 December 2010/Revised: 20 March 2011/Accepted: 23 March 2011/Published online: 3 April 2011

(C) The Author(s) 2011. This article is published with open access at Springerlink.com

\begin{abstract}
The aim of the study was to investigate health status in patients with myotonic dystrophy type 2 (DM2) and determine its relationship to pain and fatigue. Data on health status (SF-36), pain (MPQ) and fatigue (CISfatigue) were collected for the Dutch DM2 population $(n=32)$. Results were compared with those of sex- and age-matched adult-onset myotonic dystrophy type 1 (DM1) patients. In addition, we compared the obtained scores on health status of the DM2 group with normative data of the Dutch general population $(n=1742)$. Compared to DM1, the SF-36 score for bodily pain was significantly $(p=0.04)$ lower in DM2, indicating more body pain in DM2. DM2 did not differ from DM1 on any other SF-36 scales. In comparison to the Dutch population, DM2 patients reported lower scores (indicating worse clinical condition) on the physical functioning, role functioning-physical, bodily pain, general health, vitality, social functioning, and role
\end{abstract}

\section{A. A. Tieleman $(\bowtie) \cdot$ B. G. M. van Engelen}

Department of Neurology, Neuromuscular Centre Nijmegen, Radboud University Nijmegen Medical Centre, PO Box 9101, 6500 HB Nijmegen, The Netherlands

e-mail: a.tieleman@neuro.umcn.nl
A. A. Tieleman - K. M. Jenks · J. S. Kalkman - G. Borm ·
B. G. M. van Engelen
Department of Neurology, Donders Institute for Brain,
Cognition and Behavior, Radboud University
Nijmegen Medical Centre, Nijmegen, The Netherlands

K. M. Jenks · G. Borm

Department of Epidemiology, Biostatistics and HTA,

Radboud University Nijmegen Medical Centre,

Nijmegen, The Netherlands

\section{J. S. Kalkman}

Department of Medical Psychology, Radboud University

Nijmegen Medical Centre, Nijmegen, The Netherlands functioning-emotional scales ( $p<0.01$ on all scales). The difference was most profound for the physical functioning scale. In the DM2 group the severity of pain was significantly correlated with SF-36 scores for bodily pain $(p=0.003)$. Fatigue was significantly correlated with the SF-36 scores for role functioning-physical $(p=0.001)$, general health $(p=0.02)$, and vitality $(p=0.02)$. The impact of DM2 on a patients' physical, psychological and social functioning is significant and as high as in adult-onset DM1 patients. From the perspective of health-related quality of life, DM2 should not be considered a benign disease. Management of DM2 patients should include screening for pain and fatigue. Symptomatic treatment of pain and fatigue may decrease disease impact and help improve health status in DM2, even if the disease itself cannot be treated.

Keywords Myotonic dystrophy type 2 - Health status . Pain - Fatigue - Myotonic dystrophy type 1

\section{Introduction}

Myotonic dystrophy type 2 (DM2) is a dominantly inherited multisystem disease with muscle pain, weakness, myotonia, early-onset cataracts, and involvement of other organs, including the heart, brain, and gastro-intestinal system [1-3]. The genetic origin was clarified in 2001 and consists of a CCTG expansion repeat located in intron 1 of the zinc finger protein 9 (ZNF9) gene on chromosome 3q21 [4].

The phenotype and clinical course of DM2 are generally more favorable than myotonic dystrophy type 1 (DM1), the most common type of muscular dystrophy in adult life [5]. The severe congenital form in DM1 is absent in DM2, and evidence of anticipation is less striking in DM2. Muscle 
pain is more common in DM2 than in DM1, however [68]. In various neuromuscular disorders, including nondystrophic myotonic syndromes, pain has been shown to be a prominent symptom related to health status [9, 10]. Fatigue, another dominant symptom in neuromuscular diseases, is correlated with lower health status in DM1 and non-dystrophic myotonic syndromes [10, 11].

Although DM2 is a chronic disorder, up to now no studies have assessed health status in DM2 patients, where health status is defined as the impact of a disease on a patients' physical, psychological and social functioning [12]. Likewise, no data are available about the contribution of pain and fatigue on DM2 patients' health status.

In this nationwide study we investigated health status, as measured with the SF-36, in patients with genetically confirmed DM2. We chose the SF-36 because of its extensive use both in general population surveys and in patient studies, and its validated translation in Dutch [13, 14]. We also examined the presence of pain (McGill Pain Questionnaire) and fatigue (CIS-fatigue scale), and tested the hypothesis that both these two symptoms correlate with health status. Results were compared with those of sex- and age-matched adult-onset DM1 patients, and with data on health status (SF-36 scores) for the Dutch general population [15].

\section{Patients and methods}

\section{Patients}

All known Dutch DM2 patients $(n=32)$ were approached to participate and $29(91 \%)$ responded and completed the questionnaires in their home. All Dutch districts were represented in the sample and patients were treated in various hospitals throughout the Netherlands. As disease controls, we included 29 sex- and age-matched patients with adult-onset DM1. Both DM2 and DM1 patient groups were retrieved from CRAMP, the Dutch neuromuscular database [16]. Inclusion criteria were a genetically confirmed diagnosis of DM2 or DM1 at least 1 year prior to this survey and an age of 18 years and older. There were no exclusion criteria. The study was approved by the local ethical committee and all patients provided written informed consent.

Data collection

$S F-36$

Health status was assessed using the Dutch version of the SF-36 health survey. The SF-36 comprises four physical health scales (physical functioning, role limitations due to physical problems, bodily pain, and general health perception) and four mental health scales (vitality, social functioning, role limitations due to emotional problems, and mental health) [17]. Items are summed per scale and transformed into scores between 0 and 100, with higher scores indicating better function or less bodily pain [17]. The SF-36 has been used to assess health status in many different conditions, including myotonic dystrophy type 1 , inclusion body myositis and late-onset Pompe disease [11, 18, 19].

\section{$M P Q$}

Pain intensity and analgesic use were assessed with the McGill Pain Questionnaire (MPQ). The MPQ is widely used, well validated and reliable [20, 21]. Pain intensity was scored on a $100 \mathrm{~mm}$ horizontal visual analogue scale (VAS), where 0 indicates no pain and 100 indicates pain as bad as could be. The main outcome measure for pain was the magnitude of pain at the current moment.

\section{CIS-fatigue}

The presence and level of fatigue was assessed using the 'fatigue severity' subscale of the Checklist Individual Strength (CIS) [22]. The CIS measures the experience of fatigue-associated problems during the previous 2 weeks. The CIS-fatigue severity subscale contains eight items scored on a 7-point Likert scale. Scores can range between 8 and 56 with higher scores indicating higher levels of fatigue and scores of 35 or more are considered to indicate severe fatigue $[22,23]$.

Statistical analysis

Original data on health status for the Dutch general population, including SF-36 scores, age and sex, were available for analysis $(n=1,742)$ [15]. The clinimetric performance of the SF-36 in the Dutch general population has been reported previously [15]. Differences in SF36 scores between DM2 and the general population and between DM1 and the population were tested by analysis of covariance (ANCOVA) with adjustment for age and sex. Differences in SF-36 scores between DM2 and DM1 patients were tested with t-tests. Pearson correlations were computed to measure the relationship between patient characteristics and SF-36 scales. $p$ values below 0.05 were considered to be significant. Numerical variables are reported as mean \pm standard deviation (range). Categorical data are reported as number (percentage of total). 


\section{Results}

Study population

Of the 32 DM2 patients invited to participate, 29 patients (91\%) from 13 families participated in the study. Three DM2 patients did not consent and did not specify their reasons for non-participation. The disease control group existed of 29 sex- and age-matched adult-onset DM1 patients from 25 families. There were no missing values in the patient groups. The percentage of missing values for each SF-36 scale in the Dutch general population group ( $n=1,742$ ) was lowest for the social functioning and bodily pain scales $(0.4 \%, n=7$, for each) and highest for the role functioning-emotional scale $(2.9 \%, n=51)$. Demographic characteristics of the three groups are listed in Table 1. Age, sex, age of onset, disease duration and BMI did not differ significantly between the two patient groups.

\section{Health status (SF-36)}

Table 2 presents raw (unadjusted) group means on the SF-36 scales for DM2 and DM1 patients. To compare the health status of DM2 and DM1 with the general population, SF-36 scores were adjusted for age and sex. Figure 1 presents the adjusted mean SF-36 scores in DM2, DM1 and the general population.

DM2 and DM1 both scored significantly lower than the general population on physical functioning ( $p=0.001$, for both). DM2 reported a significantly lower score on the bodily pain scale (indicating a higher level of pain) than the population $(p=0.005)$, whereas DM1 did not differ from the population on the bodily pain scale $(p=0.97)$.

The scales role functioning-physical, general health, social functioning, role functioning-emotional, and vitality yielded a similar pattern of results. On each of these scales, DM2 scored significantly lower than the population $(p<0.01)$. Likewise, DM1 scored significantly lower than the population on each of these five scales $(p<0.04)$. The DM2 score on the mental health scale did not differ significantly $(p=0.06)$ from the Dutch normative sample.
Comparisons of SF-36 scales between the patient groups revealed that DM2 scored significantly lower than DM1 on bodily pain $(p=0.04)$, indicating a higher level of pain in DM2. There were no significant differences between the patient groups on the remaining SF-36 scales $(p>0.10)$.

In DM2 age was not correlated with age of onset, SF-36 scores, pain (VAS momentary and VAS maximal) scores, and CIS-fatigue. Age of onset was correlated with SF-36 bodily pain score $(r=0.39, p=0.04)$ in DM2.

In contrast, in DM1 age was correlated with age of onset ( $r=0.71, p<0.001)$, SF-36 scores physical functioning $(r=-0.50, p=0.006)$, social functioning $(r=-0.51$, $p=0.005)$, mental health $(r=-0.43, p=0.02)$, vitality $(r=-0.39, p=0.037)$, bodily pain $(r=-0.39, p=$ $0.039)$, general health $(r=-0.41, p=0.027)$ and CISfatigue score $(r=0.48, p<0.01)$. Furthermore, there was an association between age of onset and SF-36 social functioning $(r=-0.41, p=0.03)$ and mental health scores $(r=-0.41, p=0.03)$.

\section{Pain (MPQ)}

Mean VAS momentary and maximum pain scores per patient group are shown in Table 2. Twenty-three out of the 29 DM2 patients (79\%) reported pain complaints, 6 DM2 patients $(21 \%)$ reported that they had no pain at all. Eight DM2 patients (28\%) used analgesics, specifically opioids (3 patients, 10\%), NSAIDs ( 2 patients, $7 \%$ ), paracetamol (2 patients, $7 \%$ ), and amitriptyline (1 patient, $3 \%$ ).

Thirteen DM1 patients (45\%) reported having pain, the other 16 DM1 patients (55\%) reported that they had no pain at all. Seven DM1 patients (24\%) used analgesics, specifically NSAIDs (2 patients, 7\%), paracetamol (5 patients, 17\%), and amitriptyline (1 patient, 3\%).

DM2 scored significantly higher than DM1 on VAS maximum pain $(t(56)=3.07, p=0.003)$, indicating more pain in DM2. Although DM2 scored higher than DM1 on VAS momentary pain, this difference did not achieve significance $(t(56)=1.79, p=0.079)$.

In both DM2 and DM1 VAS momentary scores were correlated with VAS maximum (DM2: $r=0.70, p<$ 0.001, DM1: $r=0.81, p<0.001)$, SF-36 score bodily pain
Table 1 Characteristics of patients with DM2 and DM1, and of the general population

Data presented as mean \pm standard deviation (range) or as $\mathrm{N}(\%)$

BMI Body Mass Index

\begin{tabular}{llll}
\hline & DM2 & DM1 & $\begin{array}{l}\text { Dutch general } \\
\text { population [15] }\end{array}$ \\
\hline $\mathrm{N}$ & 29 & 29 & 1,742 \\
Age (years) & $53.2 \pm 12.1(28-71)$ & $52.8 \pm 12.0(28-72)$ & $47.6 \pm 18.0(16-94)$ \\
Females & $20(69 \%)$ & $20(69 \%)$ & $761(44 \%)$ \\
BMI $\left(\mathrm{kg} / \mathrm{m}^{2}\right)$ & $25.1 \pm 3.8(18.8-36.0)$ & $25.7 \pm 3.5(16.5-30.9)$ & - \\
Age of onset (years) & $35.9 \pm 13.4(12-67)$ & $37.2 \pm 13.7(15-61)$ & - \\
Disease duration & $17.3 \pm 15.0(2-57)$ & $17.0 \pm 9.9(1-38)$ & - \\
\hline
\end{tabular}


Table 2 Mean scores of DM2 patients and DM1 patients on the outcome measures
Data presented as mean (SD) or $\mathrm{N}(\%)$

\begin{tabular}{llcc}
\hline & DM2 & DM1 & $p$ value \\
& & & DM2-DM1 \\
\hline Health status (SF-36) & & & \\
Physical health & $38.4(27.9)$ & $50.7(28.6)$ & 0.11 \\
$\quad$ Physical functioning & $37.1(35.7)$ & $50.9(41.4)$ & 0.18 \\
$\quad$ Role functioning-physical & $57.6(24.6)$ & $73.1(31.6)$ & $\mathbf{0 . 0 4}$ \\
Bodily pain & $42.4(23.1)$ & $41.7(19.7)$ & 0.90 \\
General health & & & \\
Mental health & $45.9(23.0)$ & $39.1(16.0)$ & 0.20 \\
Vitality & $69.1(29.5)$ & $70.0(24.7)$ & 0.90 \\
$\quad$ Social functioning & $58.5(44.3)$ & $65.4(43.2)$ & 0.55 \\
Role functioning-emotional & $69.7(22.9)$ & $68.0(20.8)$ & 0.77 \\
Mental health & & & \\
Pain (MPQ) & $23(79.3)$ & $13(44.8)$ & $\mathbf{0 . 0 1 4}$ \\
Pain (\%) & $23.6(22.5)$ & $13.1(21.8)$ & 0.079 \\
VAS-momentary pain (mm) & $56.3(34.9)$ & $27.8(36.0)$ & $\mathbf{0 . 0 0 3}$ \\
VAS-maximum pain (mm) & $19(65.5)$ & $25(86.2)$ & 0.066 \\
Fatigue (CIS) & $38.7(13.1)$ & $44.3(8.7)$ & 0.058 \\
Severe fatigue (\%) & & &
\end{tabular}

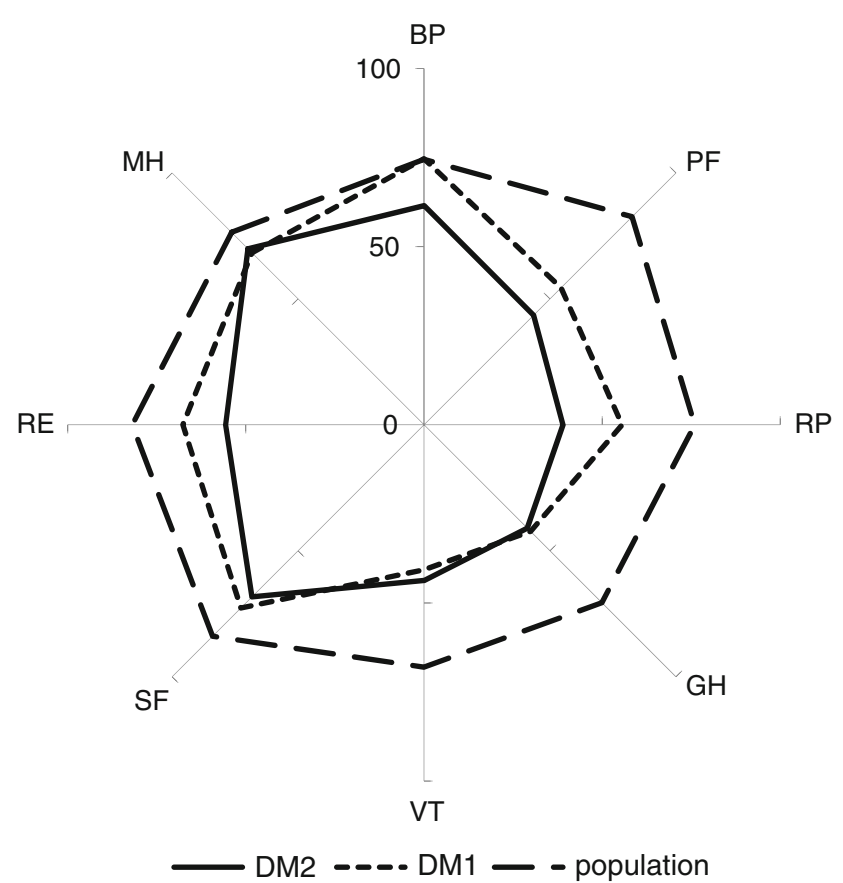

Fig. 1 Health status in Dutch myotonic dystrophy type 2 patients compared to the Dutch general population. Solid line DM2 $(n=29)$. Dotted line DM1 $(n=29)$. Dashed line general population $(n=1,742)$. Values are mean scores for SF-36 scales, adjusted for age and sex. The center of the graph represents the lowest possible score on each scale. $B P$ bodily pain, $P F$ physical functioning, $R P$ role functioning-physical, $G H$ general health, $V T$ vitality, $S F$ social functioning, $R E$ role functioning-emotional, $M H$ mental health
(DM2: $r=-0.40, p=0.03$, DM1: $r=-0.71, p<0.01$ ) and CIS-fatigue score (DM2: $r=0.39, p=0.04, \mathrm{DM} 1$ : $r=0.43, p=0.02)$. In DM1 VAS momentary scores also correlated with SF-36 score social functioning $(r=-0.51$, $p<0.01)$ and role functioning-emotional $(r=-0.40$, $p=0.03)$.

\section{Fatigue (CIS-fatigue)}

Mean fatigue scores per patient group are shown in Table 2. Nineteen of the 29 DM2 patients $(66 \%)$ and 25 out of the 29 DM1 patients $(86 \%)$ reported severe fatigue. Although there was a higher average level of fatigue in DM1 patients in comparison to DM2, this difference did not achieve significance $(p=0.059)$.

In DM2 CIS-fatigue scores were correlated with SF-36 scores role functioning-physical $(r=-0.58, p=0.001)$, vitality $(r=-0.44, p=0.016)$ and general health $(r=$ $-0.42, p=0.02)$, as well as with VAS maximum $(r=0.70, p<0.001)$. In DM1 CIS-fatigue scores were significantly correlated with all of the SF-36 scales except for the scale mental health (Table 3).

\section{Discussion}

In this nationwide study we found that DM2 patients scored significantly lower than the Dutch general population on 


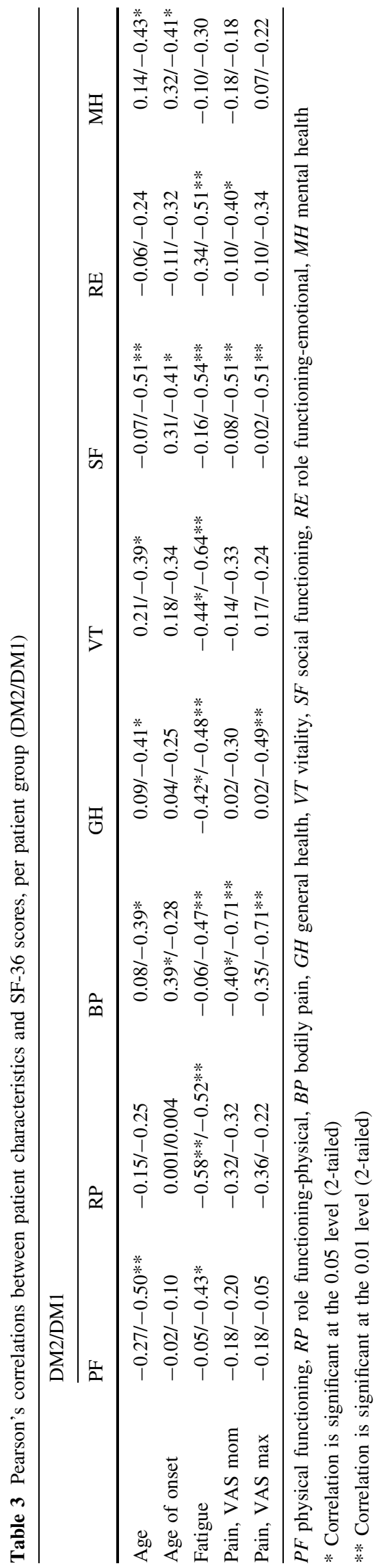

seven of the eight physical and mental health scales of the SF-36. SF-36 scores in DM2 were comparable to those of adult-onset DM1 patients on all SF-36 scales except for bodily pain scale. DM2 scored significantly lower than DM1 on the bodily pain scale, indicating more body pain in DM2. These results demonstrate that DM2 has a high disease impact on physical as well as on mental health functioning. This impact is at least comparable to that of adultonset DM1. Until now, DM2 was considered a benign disease, mainly because of the more favorable phenotype and clinical course of DM2 in comparison to DM1 [5]. However, this cross-sectional study clearly demonstrates that the symptoms of DM2 greatly impact patients' selfreported health status.

Although DM2 and DM1 are usually considered neuromuscular disorders, the results of our study reflect their multisystem character. The low scores on both physical and mental health scales in the DM2 and DM1 patients in comparison to the general population are in contrast with results from previous studies on health status in more restricted neuromuscular disorders, including non-dystrophic myotonias, inclusion body myositis, late-onset Pompe disease, and immune-mediated polyneuropathies [10, 18, 19, 24]. In these neuromuscular diseases, although low scores were found on the physical health scales, mental health scores remained relatively high. Preserved high mental scores in chronic disorders have been explained by the fact that patients' limitations in daily activities develop over a long period. Over time, patients may adapt to their situation and adjust their expectations, priorities and even redefine concepts related to mental health aspects of quality of life, leading to the so called response shift [25, 26]. It may well be possible that in DM2 and DM1, in which cognitive impairment such as frontal lobe dysfunction and an avoidant personality trait have been reported, adaptive coping behavior is reduced or even not present, in contrast to other disabling neuromuscular disorders [27, 28].

Cognition in terms of overall intelligence does not seem to be impaired in DM2 [29]. The ability to complete this self report questionnaire in a reliable and valid manner was, therefore, present in DM2 patients. In addition, all DM2 and DM1 patients who participated in the current study had been diagnosed at least 1 year prior to the study, so it is unlikely that the observed lower scores on vitality, social functioning, and role limitations due to emotional problems could be attributed to a reactive depression. Our health status results in DM1 are corroborated by previous research in which health status was measured with the SF36 in a group of 322 adult-onset DM1 patients [11]. Aside from a difference in age between the present DM1 group and the one reported by Kalkman (mean age: 52.8 years vs. 43.0 years respectively) results are comparable; all physical and mental health scores, except for the score of bodily 
pain, were lower than those of the general population. Pain was not evaluated in that study, but fatigue was correlated with a lower reported health status in physical and social functioning.

Compared to the general population, the differences in SF-36 scores of DM2 patients was most profound for the physical functioning scale [adjusted mean score of DM2 patients $43.5( \pm 21.1)$ vs. $82.6( \pm 19.7)$ of the general population]. The DM2 score on the mental health scale was the only SF-36 score not significantly $(p=0.06)$ lower compared to the Dutch normative data [adjusted mean 69.9 $( \pm 18.6)$ vs. $76.4( \pm 17.4)$ of the general population]. However, the outcome in DM2 is comparable to SF-36 mental health scores of two other samples with clearly defined chronic health conditions, namely migraine and cancer patients (SF-36 mental health score 72.0 and 68.0 respectively) [15].

In DM2, age did not correlate with any of the SF-36 scales. This lack of correlation between age and health status may be an indication that the symptoms of DM2 are relatively stable over the lifespan. There was a positive correlation between age of onset and the bodily pain scale of the SF-36, indicating more bodily pain in patients whose symptoms began at a younger age and conversely, less bodily pain in patients whose symptoms began at an older age. This implies that there may be a tendency for pain to worsen over time in DM2. However, future research should investigate these associations in the context of a prospective design.

In contrast, in DM1, age was strongly associated with age of onset. This strong correlation, showing that older patients in this sample had a later age of onset and younger patients had an early age of onset, underscores the shortened life expectancy associated with DM1. Age in DM1 was highly associated with more impairments in functioning and more body pain $(p<0.04$ on six scales), underscoring the progressive nature of this disease. These DM1 results are corroborated by previous research of us and others. In the previously mentioned study on health status in DM1 patients, similar associations were found between age and SF-36 scores [11]. As also that study had a cross-sectional design, the need for future longitudinal research exits. In contrast, recent data in nondystrophic myotonic syndromes showed no correlation between age and SF-36 scores, indicating that age did not appear to play a role in health status [10]. Taken together, these results again underscore the progressive nature and shortened life expectancy in DM1 in comparison to DM2 and non-dystrophic myotonic syndromes.

In DM2, age did not correlate with pain, as measured with VAS, or fatigue, as measured with CIS, again indicating that the symptoms of DM2 may remain somewhat stable over the lifespan. There was a significant correlation in DM2 between increased pain and lower levels of mental health. Also, fatigue was negatively correlated to the scales of vitality, general health and role functioning-physical. Of course, one cannot infer causality on the basis of correlations alone. However, both pain and fatigue appear to be related to a decrease of health status in DM2. Pain is a chronic symptom in DM2 and the observed association between pain and mental health underscores the need to identify and provide effective pain treatments for DM2 patients [6].

Some mention should be made of potential limitations of this study. First, in eight families with DM2, several members were evaluated. Shared genetic cofactors or recognized behavioral biasing could have influenced pain, and data on physical, psychological and social functioning. Second, because DM2 is, in general, more likely to be under diagnosed than DM1, the participating DM2 patients may be more representative of the severe end of the DM2 disease spectrum, leading to lower physical and functional scores. Third, by studying the Dutch DM2 population, this study does not take potential cultural differences (e.g. the Netherlands vs. United States) into account. However, evidence suggests that cultural differences do not play a role in health status in neuromuscular disorders [19, 30]. Fourth, despite the solidity of our nationwide findings, it should be noted that we did not evaluate the clinimetric properties of the SF-36 in these specific patient populations, an omission that future research in this area could address.

Despite these limitations, the comprehensiveness of the SF-36 may help to increase physicians' awareness, by providing information on health status in patients with DM2, besides the traditional investigation of symptoms, signs and laboratory studies.

In summary, the current study shows the impact of DM2 on a patients' physical, psychological and social functioning is significant and as high as in adult-onset DM1 patients. From the perspective of health-related quality of life, DM2 should, therefore, not be considered a benign disease. Pain and fatigue are correlated to a lower reported health status. Management of DM2 patients should include screening for pain and fatigue. Symptomatic treatment of these two symptoms may decrease disease impact and improve health status in DM2, even if the disease itself cannot be treated. There remains a need for prospective follow-up studies assessing the natural course of DM2 in relation to health status, fatigue and pain.

Acknowledgments We gratefully acknowledge Prof. Neil Aaronson for health population material used in this study.

Conflict of interest The authors have reported no conflicts of interest. 
Open Access This article is distributed under the terms of the Creative Commons Attribution Noncommercial License which permits any noncommercial use, distribution, and reproduction in any medium, provided the original author(s) and source are credited.

\section{References}

1. Harper PS, van Engelen BGM, Eymard B et al (2004) Myotonic dystrophy: present management, future therapy. Oxford University Press, New York

2. Meola G, Sansone V (2007) Cerebral involvement in myotonic dystrophies. Muscle Nerve 6:294-306

3. Tieleman AA, van Vliet J, Jansen JB et al (2008) Gastrointestinal involvement is frequent in myotonic dystrophy type 2 . Neuromuscul Disord 18:646-649

4. Liquori CL, Ricker K, Moseley ML et al (2001) Myotonic dystrophy type 2 caused by a CCTG expansion in intron 1 of ZNF9. Science 293:864-867

5. Udd B, Meola G, Krahe R et al (2006) 140th ENMC international workshop: myotonic dystrophy DM2/PROMM and other myotonic dystrophies with guidelines on management. Neuromuscul Disord 16:403-413

6. George A, Schneider-Gold C, Zier S et al (2004) Musculoskeletal pain in patients with myotonic dystrophy type 2. Arch Neurol 61:1938-1942

7. Turner C, Hilton-Jones D (2010) The myotonic dystrophies: diagnosis and management. J Neurol Neurosurg Psychiatry $81: 358-367$

8. Jensen MP, Hoffman AJ, Stoelb BL et al (2008) Chronic pain in persons with myotonic dystrophy and facioscapulohumeral dystrophy. Arch Phys Med Rehabil 89:320-328

9. Jensen MP, Abresch RT, Carter GT et al (2005) Chronic pain in persons with neuromuscular disease. Arch Phys Med Rehabil 86:1155-1163

10. Trip J, de Vries J, Drost G et al (2009) Health status in nondystrophic myotonias: close relation with pain and fatigue. J Neurol 256:939-947

11. Kalkman JS, Schillings ML, van der Werf SP et al (2005) Experienced fatigue in facioscapulohumeral dystrophy, myotonic dystrophy, and HMSN-I. J Neurol Neurosurg Psychiatry 76:1406-1409

12. De Vries J (2001) Quality of life assessment. In: Vingerhoets AJJM (ed) Assessment in behavioral medicine. Brunner-Routledge, Hove, pp 353-370

13. Wagner AK, Gandek B, Aaronson NK et al (1998) Cross-cultural comparisons of the content of SF-36 translations across 10 countries: results from the IQOLA project. International quality of life assessment. J Clin Epidemiol 51:925-932

14. Gandek B, Ware JE Jr (1998) Methods for validating and norming translations of health status questionnaires: the IQOLA Project approach. International quality of life assessment. J Clin Epidemiol 51:953-959
15. Aaronson NK, Muller M, Cohen PD et al (1998) Translation, validation, and norming of the Dutch language version of the SF36 Health Survey in community and chronic disease populations. J Clin Epidemiol 51:1055-1068

16. van Engelen BG, van Veenendaal H, van Doorn PA et al (2007) The Dutch neuromuscular database CRAMP (Computer Registry of All Myopathies and Polyneuropathies): development and preliminary data. Neuromuscul Disord 17:33-37

17. Ware JE Jr, Sherbourne CD (1992) The MOS 36-item short-form health survey (SF-36). Conceptual framework and item selection. Med Care 30:473-483

18. Sadjadi R, Rose MR, Muscle Study Group (2010) What determines quality of life in inclusion body myositis? J Neurol Neurosurg Psychiatry 81:1164-1166

19. Hagemans ML, Janssens AC, Winkel LP et al (2004) Late-onset Pompe disease primarily affects quality of life in physical health domains. Neurology 63:1688-1692

20. Vanderiet K, Adriaensen H, Carton H et al (1987) The McGill pain questionnaire constructed for the Dutch language (MPQDV). Preliminary data concerning reliability and validity. Pain 30:395-408

21. Van der Kloot WA, Oostendorp RA, van der Meij J et al (1995) The Dutch version of the McGill pain questionnaire: a reliable pain questionnaire. Ned Tijdschr Geneeskd 139:669-673

22. Vercoulen JH, Swanink CM, Fennis JF et al (1994) Dimensional assessment of chronic fatigue syndrome. J Psychosom Res 38:383-392

23. Prins JB, Bleijenberg G, Bazelmans E et al (2001) Cognitive behavior therapy for chronic fatigue syndrome: a multicentre randomised controlled trial. Lancet 17:841-847

24. Merkies ISJ, Schmitz PIM, van der Meche FGA et al (2002) Quality of life complements traditional outcome measures in immune-mediated polyneuropathies. Neurology 59:84-91

25. Carver CS, Scheier MF (2000) Scaling back goals and recalibration of the affect system are processes in normal adaptive selfregulation: understanding 'response shift' phenomena. Soc Sci Med 50:1715-1722

26. Kempen GI, Ormel J, Brilman EI et al (1997) Adaptive responses among Dutch elderly: the impact of eight chronic medical conditions on health-related quality of life. Am J Public Health $87: 38-44$

27. Meola G, Sansone V, Perani D et al (2003) Executive dysfunction and avoidant personality trait in myotonic dystrophy type 1 (DM1) and in proximal myotonic myopathy (PROMM/DM-2). Neuromuscul Disord 13:813-821

28. Meola G, Sansone V, Perani D et al (1999) Reduced cerebral blood flow and impaired visual-spatial function in proximal myotonic myopathy. Neurology 53:1042-1050

29. Meola G, Sansone V (2007) Cerebral involvement in myotonic dystrophies. Muscle Nerve 36:294-306

30. Jenkinson C (1999) Comparison of UK and US methods for weighting and scoring the SF-36 summary measures. J Public Health Med 21:372-376 